\title{
QTL analysis of photoperiod sensitivity in common buckwheat by using markers for expressed sequence tags and photoperiod-sensitivity candidate genes
}

\author{
Takashi Hara' ${ }^{1)}$ Hiroyoshi Iwata ${ }^{2}$, Kazutoshi Okuno(1), Katsuhiro Matsui ${ }^{3)}$ and Ryo Ohsawa*1) \\ 1) Graduate School of Life and Environmental Sciences, University of Tsukuba, 1-1-1 Tennondai, Tsukuba, Ibaraki 305-8572, Japan \\ 2) Graduate School of Agriculture and Life Science, The University of Tokyo, 1-1-1 Yayoi, Bunkyo, Tokyo 113-8657, Japan \\ 3) National Agricultural and Food Research Organization, National Agricultural Research Center for Kyushu Okinawa Region, 2421 \\ Suya, Koushi, Kumamoto 861-1192, Japan
}

\begin{abstract}
Photoperiod sensitivity is an important trait related to crop adaptation and ecological breeding in common buckwheat (Fagopyrum esculentum Moench). Although photoperiod sensitivity in this species is thought to be controlled by quantitative trait loci (QTLs), no genes or regions related to photoperiod sensitivity had been identified until now. Here, we identified QTLs controlling photoperiod sensitivity by QTL analysis in a segregating $\mathrm{F}_{4}$ population $(n=100)$ derived from a cross of two autogamous lines, 02AL113(Kyukei SC2)LH.self and C0408-0 RP. The $\mathrm{F}_{4}$ progenies were genotyped with three markers for photoperiodsensitivity candidate genes, which were identified based on homology to photoperiod-sensitivity genes in Arabidopsis and 76 expressed sequence tag markers. Among the three photoperiod-sensitivity candidate genes ( $F e C C A 1, F e E L F 3$ and $F e C O L 3$ ) identified in common buckwheat, FeELF3 was associated with photoperiod sensitivity. Two EST regions, Fest_L0606_4 and Fest_L0337_6, were associated with photoperiod sensitivity and explained $20.0 \%$ and $14.2 \%$ of the phenotypic variation, respectively. For both EST regions, the allele from 02AL113(Kyukei SC2)LH.self led to early flowering. An epistatic interaction was also confirmed between Fest_L0606_4 and Fest_L0337_6. These results demonstrate that photoperiod sensitivity in common buckwheat is controlled by a pathway consisting of photoperiod-sensitivity candidate genes as well as multiple gene action.
\end{abstract}

Key Words: common buckwheat, EST markers, photoperiod-sensitivity candidate genes, photoperiod sensitivity, QTL analysis.

\section{Introduction}

Common buckwheat (Fagopyrum esculentum Moench, $2 n=16$ ), a pseudocereal, has been widely cultivated in Asia, Europe and North America. In Japan, common buckwheat has been cultivated throughout most of the country, with cultivars classified into three ecotypes: summer, intermediate, and late-summer. The late-summer cultivars are cultivated in low-latitude regions where photoperiod is around 12 to $14 \mathrm{~h}$ during the cropping season, and their flowering is generally sensitive to photoperiod. The summer cultivars are cultivated in high-latitude regions where photoperiod is around 14.5 to $15.5 \mathrm{~h}$ during the cropping season (summer crop season), and they are generally insensitive to photoperiod. Intermediate cultivars show moderate photoperiod sensitivity (Namai 1990). Common buckwheat is considered to be a short-day plant, with the threshold between flowering and non-flowering at a photoperiod of about $14.5 \mathrm{~h}$

Communicated by T. Hayashi

Received April 27, 2011. Accepted September 16, 2011.

*Corresponding author (e-mail: osawaryo@sakura.cc.tsukuba.ac.jp)
(Nagatomo 1961). Under long-day conditions, (i.e., photoperiod longer than $14.5 \mathrm{~h}$ ), late-summer cultivars showed wide variation in flowering time, which resulted in low yield because of the increased incidence of malformed flowers and the decrease in ripening rate in the late-flowering individuals (Nagatomo 1961, Nakamura and Nakayama 1950, Sugawara 1958). The intermediate and summer cultivars are considered to have been derived from the late-summer cultivars through both natural and artificial selection (Matano and Ujihara 1979). Minami and Namai (1986a, 1986b) suggested that summer cultivars differentiated from latesummer cultivars through the selection of early-flowering plants under long-day conditions; this selection was part of the domestication of buckwheat for climatic adaptation to the northern part of Japan.

Iwata et al. (2005) reported that allelic richness (i.e., the degree of polymorphism of genetic markers) has decreased in Japanese common buckwheat varieties cultivated in highlatitude regions during selection for early flowering time, and they suggested that the decrease of allelic richness at neutral loci was caused by bottleneck effects caused by directional selection based on photoperiod sensitivity. Ohsawa 
(1997) reported that the heritability of flowering period was high (95.1\%), suggesting that photoperiod sensitivity was a key feature of adaptation and differentiation in common buckwheat. Photoperiod-sensitivity genes, however, have not been identified in common buckwheat.

Minami (1985) suggested that photoperiod sensitivity in common buckwheat was controlled by a number of genes. In several crops, photoperiod sensitivity is considered to be inherited quantitatively (Putterill et al. 2004, Yano et al. 2001). Genetic regulation of flowering induced by photoperiod has been studied in Arabidopsis and rice (Oryza sativa L.), and many genes related to photoperiod sensitivity have been detected as quantitative trait loci (QTLs) and isolated by positional cloning (Corbesier and Coupland 2005, Hayama and Coupland 2003). These genes compose the photoperiod-sensitivity pathway (Putterill et al. 2004), and have been confirmed among many species as orthologous genes (Hayama et al. 2002, 2003, Hayama and Coupland 2003, Kojima et al. 2002, Liu et al. 2001a, Nemoto et al. 2003, Yano et al. 2000). Moreover, photoperiod sensitivity in both long-day and short-day plants appears to be controlled by a common mechanism (Putterill et al. 2004). Based on this information, we hypothesized that photoperiod sensitivity of common buckwheat would also be controlled by the action of multiple genes orthologous to those of the photoperiod-sensitivity pathways in other species.

To avoid the complex genetic patterns caused by the allogamous (cross-pollinating) reproductive system of most common buckwheat, we used autogamous (self-pollinating) lines showing differences in photoperiod sensitivity for simplicity of analysis. We used expressed sequence tag (EST) markers derived from cDNAs of candidate photoperiod-sensitivity genes to detect photosensitivity QTLs most efficiently.

In the present study, QTLs related to photoperiod sensitivity in common buckwheat were identified in the following three steps: (1) evaluation of variation in photoperiod sensitivity between two autogamous parental lines, (2) linkage map development using EST markers, and (3) QTL analysis for photoperiod sensitivity.

\section{Materials and Methods}

\section{Photoperiod sensitivity of parental lines}

For detecting QTLs related to photoperiod sensitivity, we employed two parental lines, 02AL113(Kyukei SC2)LH.self (KYU) and C0408-0RP (CAN). KYU is an autogamous line bred at the National Agricultural Research Center for Kyushu Okinawa Region, and CAN is an autogamous line bred at Kade Research Ltd. (Canada). In general, differences in photoperiod sensitivity between these two lines was visible under photoperiods longer than $14.5 \mathrm{~h}$. We used two photoperiod conditions, a natural long-day condition (day length of $\sim 14.5 \mathrm{~h}$ ) and an artificial long-day condition $(15.5 \mathrm{~h})$, to detect the difference in photoperiod sensitivity between KYU and CAN. For the natural long-day condition, 60 seeds each of KYU and CAN were sowed by 12 seeds per one planter $(19 \times 59 \times 16 \mathrm{~cm})$ and cultivated in growth cabinet without supplemental light at the University of Tsukuba. For the artificial long-day condition, 12 seeds each of KYU and CAN were sowed in a planter and cultivated in a growth cabinet with day length of $15.5 \mathrm{~h}$. Metal halide lamps (MLBOC400C-U) (Mitsubishi Electric Corp., Tokyo, Japan) were used to control the day length at artificial longday condition.

The investigation of photoperiod sensitivity was performed as follows. The dates of cotyledon development and of the first flower flowering were recorded for each individual. The number of days from the expansion of cotyledons to the first flower flowering was defined as days-to-flowering, which was used as a measure of the photoperiod sensitivity of each individual. The measurements were performed every day until 100 days after sowing. At 100 days after sowing, individuals that had not yet flowered were classified as 'nonflowering' and these were excluded from the following analyses. To evaluate differences in photoperiod sensitivity between the populations, we performed analysis of variance (ANOVA) and $t$ tests based on the days-to-flowering under each photoperiod condition by using the program JMP 6.0 (SAS Institute Inc., NC, USA).

cDNA library construction and search for photoperiodsensitivity candidate gene regions

We developed sets of 362 cDNA clones derived from inflorescences and 1920 cDNA clones derived from leaves. 362 cDNA clones derived from inflorescences was provided by the Niigata University of Pharmacy and Applied Life Sciences. To develop 1920 cDNA clones derived from leaves, we used 'Miyazakizairai' (late-summer cultivar) for plant material and sampled leaves six times at 4-h intervals from individuals that were either early flowering or non-flowering under the $15.5 \mathrm{~h}$ day-length condition because it is clarified that amount of photoperiod-sensitivity genes expression in Arabidopsis was changed over time. Total RNA was extracted by using RNeasy Plant Mini Kit (Qiagen Inc., CA, USA) following manufacturer's instructions. cDNA library was directionally constructed (5' SfiIA, 3' SfiIB) in pDNR-LIB using the Creator SMART cDNA Library Construction Kit (Clontech Laboratories, Inc., CA, USA) and size-fractionated by using CHROMA SPIN+TE-1000 (Clontech Laboratories, Inc.). Libraries were manually arrayed in 96-well microtiter plates. Glycerol stocks of overnight cultures were prepared in 96-well format. Plasmid DNAs were extracted and BigDye Terminator (Life Technologies Corp., NY, USA) cycle sequenced on ABI PRISM 3730xl Genetic Analyzer (Life Technologies Corp.) by using conventional procedures and the following primers: M13 forward primer (5'GTAAAACGACGGCCAGT-3') and M13 reverse primer (5'-AAACAGCTATGACCATGTTCA). Vector sequences and low quality regions were trimmed from EST sequences manually before removing overlapping regions by using BLASTN, and then 863 clones (139 inflorescence-derived clones, designated as the Fest_F group, and 724 leaf-derived 
clones, designated as the Fest_L group) were used for further analysis. To search for photoperiod-sensitivity candidate gene regions, a homology search against previously reported sequences to Arabidopsis was performed by using tBLASTX. Matches were considered to be significant when the smallest sum probability $(p)$ was less than 0.0001 and bit scores were greater than 100 .

\section{Development of the mapping population}

We developed a mapping population consisting of $100 \mathrm{~F}_{4}$ lines derived by single-seed descent from a cross between KYU as the female parent and CAN as the male parent. Because we observed stunted growth in the $\mathrm{F}_{5}$ generation, we used the $\mathrm{F}_{4}$ generation as the population for linkage mapping and QTL analysis.

Total DNA samples of both parental lines and the $\mathrm{F}_{4}$ lines were extracted by the following method. About $100 \mathrm{mg}$ leaf tissue was mixed with $500 \mu$ lysis buffer $(0.3 \%$ sodium dodecyl sulfate, $20 \mathrm{mM}$ Tris- $\mathrm{HCl}\left(\mathrm{pH} 8.0\right.$ at $\left.25^{\circ} \mathrm{C}\right), 5 \mathrm{mM}$ EDTA, $400 \mathrm{mM} \mathrm{NaCl}$ ), and incubated at $65^{\circ} \mathrm{C}$ for $10 \mathrm{~min}$ with $5 \mu \mathrm{l}$ of $10 \mathrm{mg} / \mathrm{ml}$ Proteinase K (Wako Pure Chemical Ind., Ltd., Osaka, Japan) and $100 \mathrm{mg} / \mathrm{ml}$ RNase A (Qiagen Inc.). The DNA was purified with chloroform-octanol (24:1) and precipitated with isopropanol. The pellet was washed with $70 \%$ ethanol and resuspended in $100 \mu \mathrm{l}$ TrisEDTA buffer.

\section{Marker development and linkage map construction}

Primer sets corresponding to three candidate genes (identified in the homology search described above) and randomly selected 170 ESTs (78 ESTs from the Fest_F cDNA clones, 92 ESTs from the Fest_L cDNA clones) among 863 cDNA clones were designed by using PRIMER3 software (Supplemental Table 1). PCR was performed with candidate gene and EST primers and genomic DNA isolated from KYU and CAN as the template. The amplified products were then sequenced to check whether the target region had been amplified. PCR was performed under the following conditions: 30 ng of genomic DNA was amplified by PCR in a $30 \mu \mathrm{l}$ final reaction volume containing $6 \mu \mathrm{M}$ each of the forward and reverse primers, $6 \mathrm{mM}$ each of deoxynucleotide triphosphate (dNTPs), 10× PCR buffer, and 0.75 units of Blend Taq DNA polymerase (TOYOBO Co., Ltd., Tokyo, Japan). PCR was performed by using the Mastercycler gradient PCR system (Eppendorf Scientific Inc., Hamburg, Germany). The PCR program consisted of a preliminary denaturation step of $2 \mathrm{~min}$ at $94^{\circ} \mathrm{C}$; followed by 40 cycles of $30 \mathrm{~s}$ at $94^{\circ} \mathrm{C}$ (denaturation), $30 \mathrm{~s}$ at gradient temperatures (50 to $70^{\circ} \mathrm{C}$ ) (annealing), and $2 \mathrm{~min}$ at $72^{\circ} \mathrm{C}$ (extension); with a final extension for $7 \mathrm{~min}$ at $72^{\circ} \mathrm{C}$. PCR products were analyzed by electrophoresis in $8 \%$ acrylamide $(29: 1$ acrylamide:bis-acrylamide) gels. For sequencing, the PCR products within a single band were purified by using the QIAquick PCR purification kit (Qiagen Inc.) and then sequenced by using the BigDye Terminator ver. 3.1 Cycle Sequencing Kit (Life Technologies Corp.) and an ABI PRISM
3100 Genetic Analyzer (Life Technologies Corp.).

Primer sets that produced large differences in amplicon length (detected on $8 \%$ acrylamide gels) between parental lines were developed as EST markers. In cases where no difference in amplicon length could be detected, we searched for single-nucleotide polymorphisms (SNPs) by sequencing, and developed cleaved amplified polymorphic sequence (CAPS) markers in regions where SNPs were found within restriction-enzyme recognition sites. When no SNP could be found within the recognition sequences of restriction enzymes, derived cleaved amplified polymorphic sequence (dCAPS) markers were developed instead. The SNP searches were performed by using Phred/Phrap/Consed software (Gordon and Green 1998). The selection of restriction enzymes to detect SNPs and design of mismatch primers for dCAPS was conducted with dCAPS Finder 2.0 (Neff et al. 2002; http://helix.wustl.edu/dcaps/dcaps.html).

To detect polymorphisms of CAPS and dCAPS markers, PCR products produced by using EST primers or dCAPS primers were digested with a corresponding restriction enzyme in $12 \mu \mathrm{l}$ reaction volumes containing $10 \mu \mathrm{l}$ of the PCR products, 6 units restriction enzyme, and $1.2 \mu \mathrm{l}$ of the supplied $10 \times$ buffer. The reaction temperature was set to the recommended temperature for each restriction enzyme, and the reaction time was $3 \mathrm{~h}$. PCR and electrophoresis conditions were the same as described above.

The candidate gene and EST markers were assayed in the $\mathrm{F}_{4}$ mapping population. A framework linkage map for QTL analysis was constructed with the software JOINMAP 4.0 (Van Ooijen 2006) by using genotype data for each marker. The fit to the Mendelian segregation ratio for each marker was tested with the Chi-square test $(P<0.05)$. Markers were assigned to linkage groups with a logarithm of odds (LOD) threshold of 3.0 .

\section{QTL analysis}

The $\mathrm{F}_{4}$ population was cultivated in a growth cabinet and scored for photoperiod sensitivity under a $15.5 \mathrm{~h}$ photoperiod, as described above. QTL analysis was performed by using the software MAPQTL version 5 (Van Ooijen 2005). Interval mapping analysis was performed (Lander and Botstein 1989) to locate preliminary QTL positions on the map. Interval mapping was used to select markers significantly associated with the trait to constitute an initial set of cofactors. A backward-elimination procedure was applied to the initial set of cofactors. Only significant markers $(P<0.02)$ were used as cofactors in the multiple QTL method (MQM) (Jansen 1993, Jansen and Stam 1994) analysis for QTL detection. After the selection of cofactors, MQM analysis was performed. A 1000-permutation test was applied to each data set to establish the LOD value to be used for determining the significance $(P<0.05)$ of identified QTLs (Churchill and Doerge 1994). The phenotypic variance explained by a single QTL was calculated as the square of the partial correlation coefficient $\left(r^{2}\right)$ with the observed variable, adjusted for cofactors. When candidate gene markers 
could not be mapped, the association between each of candidate gene markers and photoperiod sensitivity was analyzed with ANOVA. To evaluate digenic interactions between pairs of QTLs, we performed two-way ANOVA for twolocus QTL genotypes, as represented by the genotypes of markers nearest QTLs These ANOVA were calculated by using the program JMP 6.0.

\section{Results}

Natural variation in photoperiod sensitivity between autogamous lines

The frequency distributions of days-to-flowering of KYU and CAN are shown in Fig. 1. Under the natural long-day condition, the days-to-flowering distributions of the KYU (range 22-38 days) and CAN (21-32 days) populations were similar (Fig. 1A), and the means (24.7 and 24.9 for KYU and CAN, respectively) were not significantly different $(P=0.681)$ (Fig. 1A). On the other hand, under the artificial long-day condition with $15.5 \mathrm{~h}$ day length, the mean daysto-flowering in KYU (mean 24.6; range 24-31 days) was significantly shorter $(P<0.0001)$ than that of CAN (mean 48.0; range 41-59 days) (Fig. 1B).

Search for photoperiod-sensitivity candidate gene regions

In tBLASTX searches against the reference sequences of proteins in Arabidopsis, three Fest_L EST regions (Fest L0268, Fest L0327 and Fest L0352) showed high homology with photoperiod-sensitivity genes in Arabidopsis. Fest_L0268 showed high homology with CONSTANS-LIKE 3 (COL3), CONSTANS-LIKE 1 (COL1) and CONSTANSLIKE 2 (COL2); Fest_L0327, with CIRCADIAN CLOCK ASSOCIATED 1 (CCA1) and LATE ELONGATED HYPOCOTYL (LHY); and Fest_L0352, with EARLY FLOWERING 3 (ELF3) (Table 1). Among the sequences with homology to multiple genes, Fest_L0268 showed the highest homology with COL3, and Fest_L0327 showed the highest homology with $C C A 1$. We considered these three EST regions as candidate genes related to photoperiod sensitivity in common buckwheat, and designated them $\mathrm{FeCOL3}, \mathrm{FeCCA1}$, and FeELF3, respectively. None of the Fest_F regions showed high homology to photoperiod genes in Arabidopsis.

Development of linkage map using EST markers

We developed primers for the three candidate genes
A

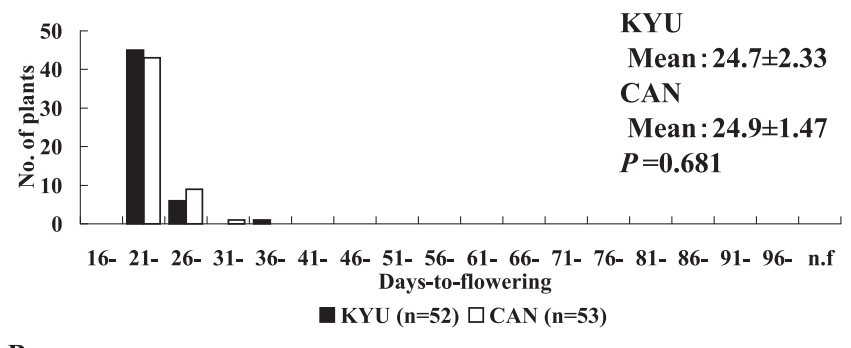

B

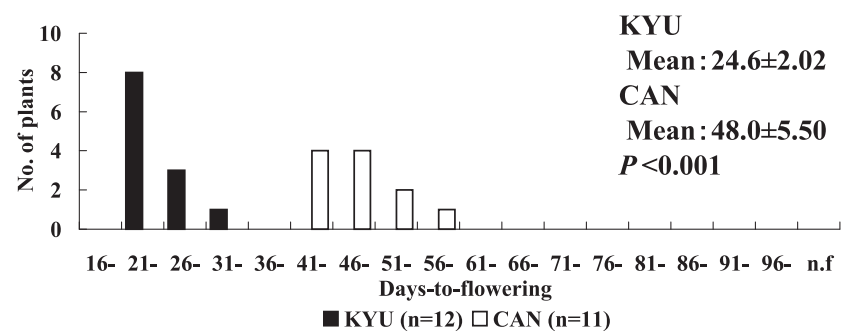

Fig. 1. Variation in days-to-flowering between the parental lines. (A) Variation in days-to-flowering of parental lines under natural long-day condition ( $\sim 14.5 \mathrm{~h}$ day length). (B) Variation in days-to-flowering of parental lines under artificial long-day condition ( $15.5 \mathrm{~h}$ day length). KYU, 02AL113(Kyukei SC2)LH.self., CAN, C0408-0 RP; $P$ value in each figure showed significance of difference by t-test.

(FeCOL3, FeCCA1 and FeELF3) and randomly selected 170 ESTs ( 78 from the Fest_F group, 92 from the Fest L group) from among the original 863 EST regions (Supplemental Tables 1 and 2 showed results of BLASTX for randomly selected 170 EST regions). Among these, we confirmed polymorphism between KYU and CAN for the three candidate gene and 115 ESTs regions. Sixteen of the 115 polymorphic EST regions included large indels, whereas the three candidate gene and 99 ESTs regions included small indels and SNPs. The 16 EST regions containing large indels were used as length markers; in addition, we developed three candidate gene and 60 SNP-based markers (39 CAPS and 21 dCAPS markers) from among the 99 ESTs markers. Information on the three candidate gene markers and 76 EST markers is shown in Table 2.

Through the linkage mapping of these markers, the positions of 63 EST markers were estimated. Nine other EST markers showed departure from Mendelian segregation ratios, and four EST markers could not be mapped (Table 2 and Fig. 2). The map consisted of 9 linkage groups (LG)

Table 1. Confirmed sequences showing high homology with photoperiod-sensitivity genes in Arabidopsis

\begin{tabular}{llrrr}
\hline \hline EST region & & Gene ID & Bit score & E-value \\
\hline Fest_L0268 & COL3 (CONSTANS-LIKE 3); protein binding / transcription factor/ zinc ion binding & 817016 COL3 & 124 & $4.00 \mathrm{E}-29$ \\
& COL1 (constans-like 1); transcription factor/ zinc ion binding & 831442 COL1 & 112 & $2.00 \mathrm{E}-25$ \\
& COL2 (constans-like 2); transcription factor/ zinc ion binding & 821298 COL2 & 112 & $2.00 \mathrm{E}-25$ \\
Fest_L0327 & CCA1 (CIRCADIAN CLOCK ASSOCIATED 1); DNA binding / transcription activator/ & 819296 CCA1 & 142 & $4.00 \mathrm{E}-34$ \\
& transcription factor/ transcription repressor & & & \\
& LHY (LATE ELONGATED HYPOCOTYL); DNA binding / transcription factor & 839341 LHY & 137 & $5.00 \mathrm{E}-33$ \\
Fest_L0352 & ELF3 (EARLY FLOWERING 3); protein C-terminus binding / transcription factor & 817134 ELF3 & 104 & $1.00 \mathrm{E}-22$ \\
\hline
\end{tabular}




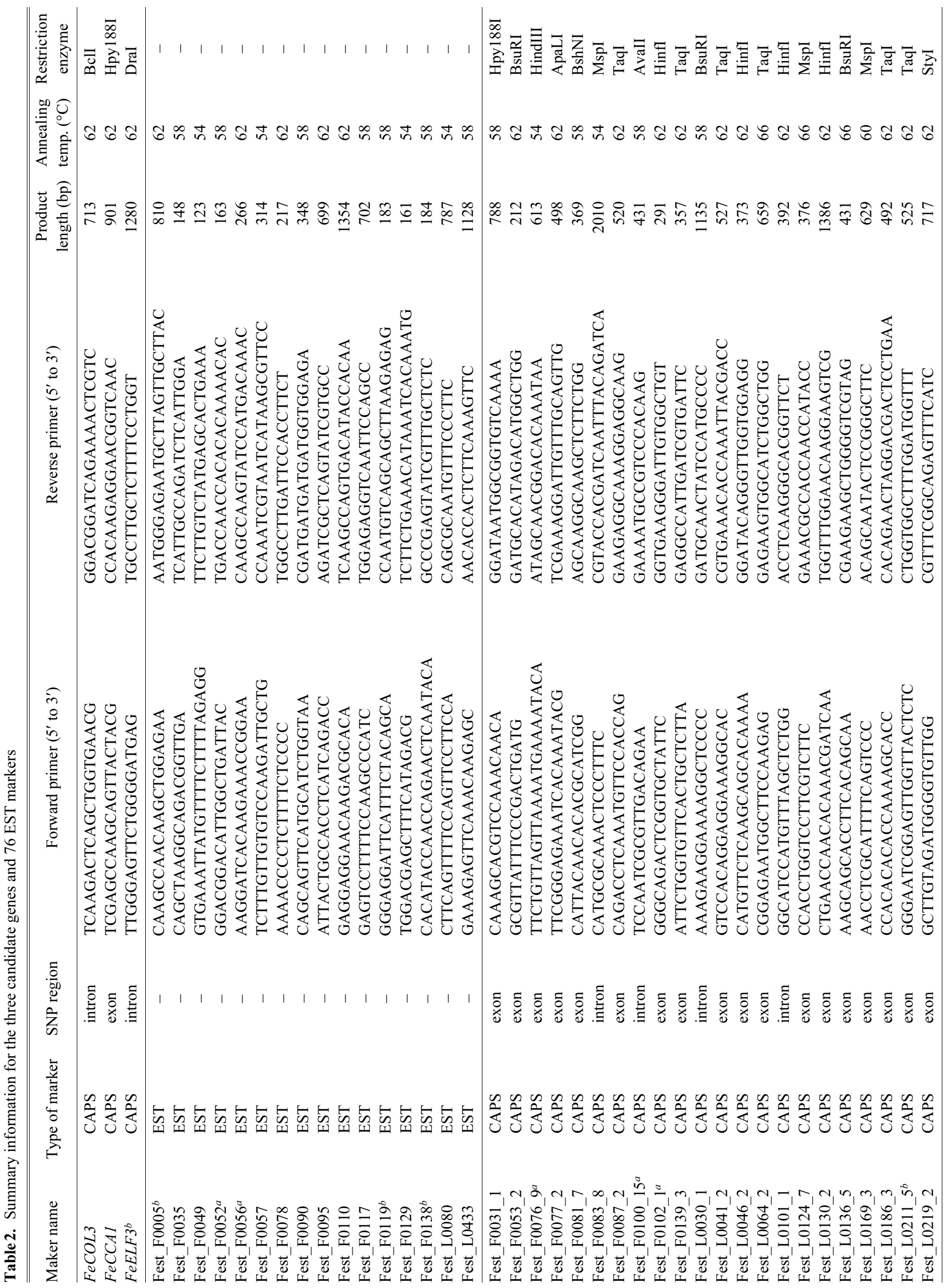




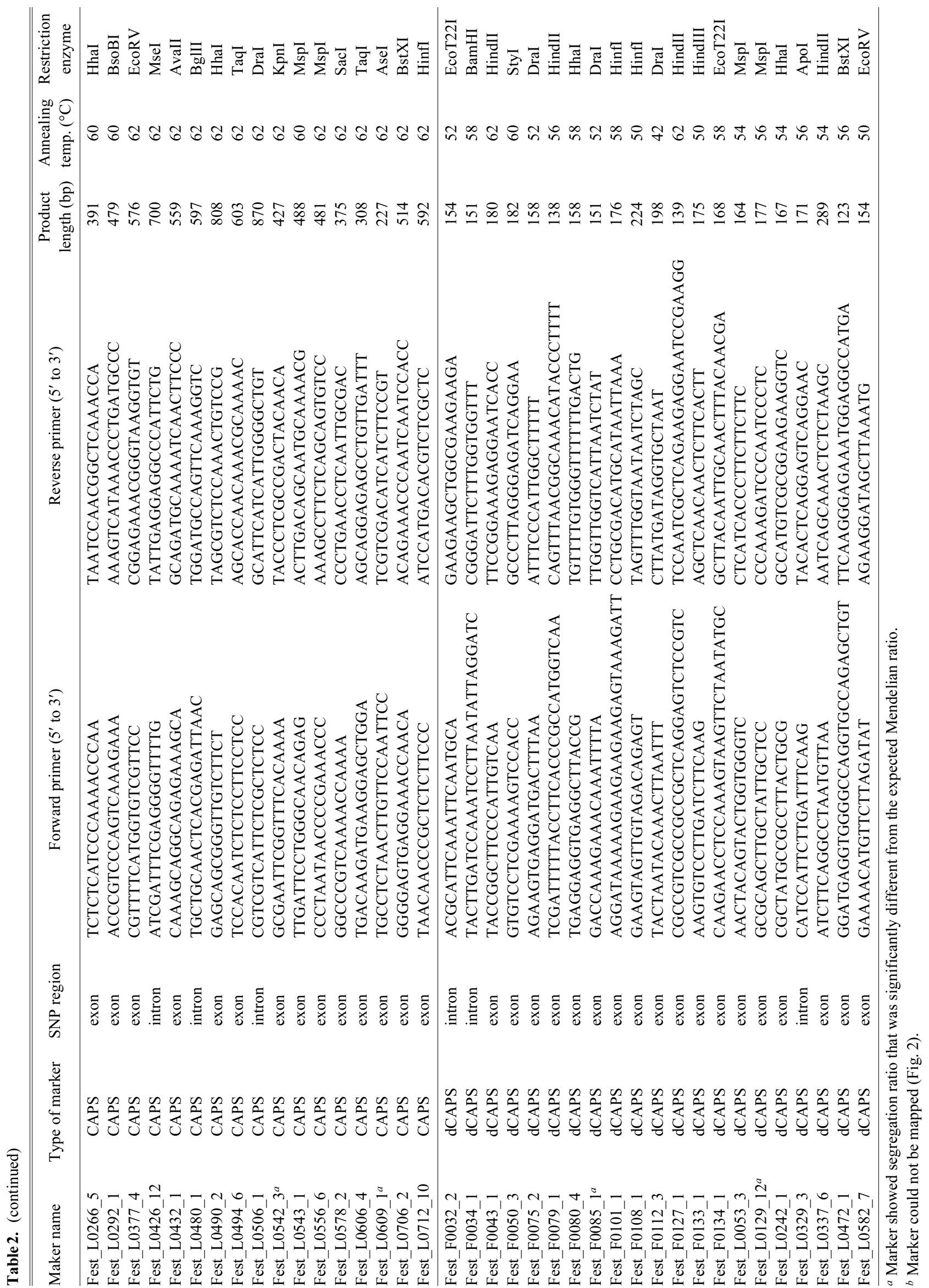




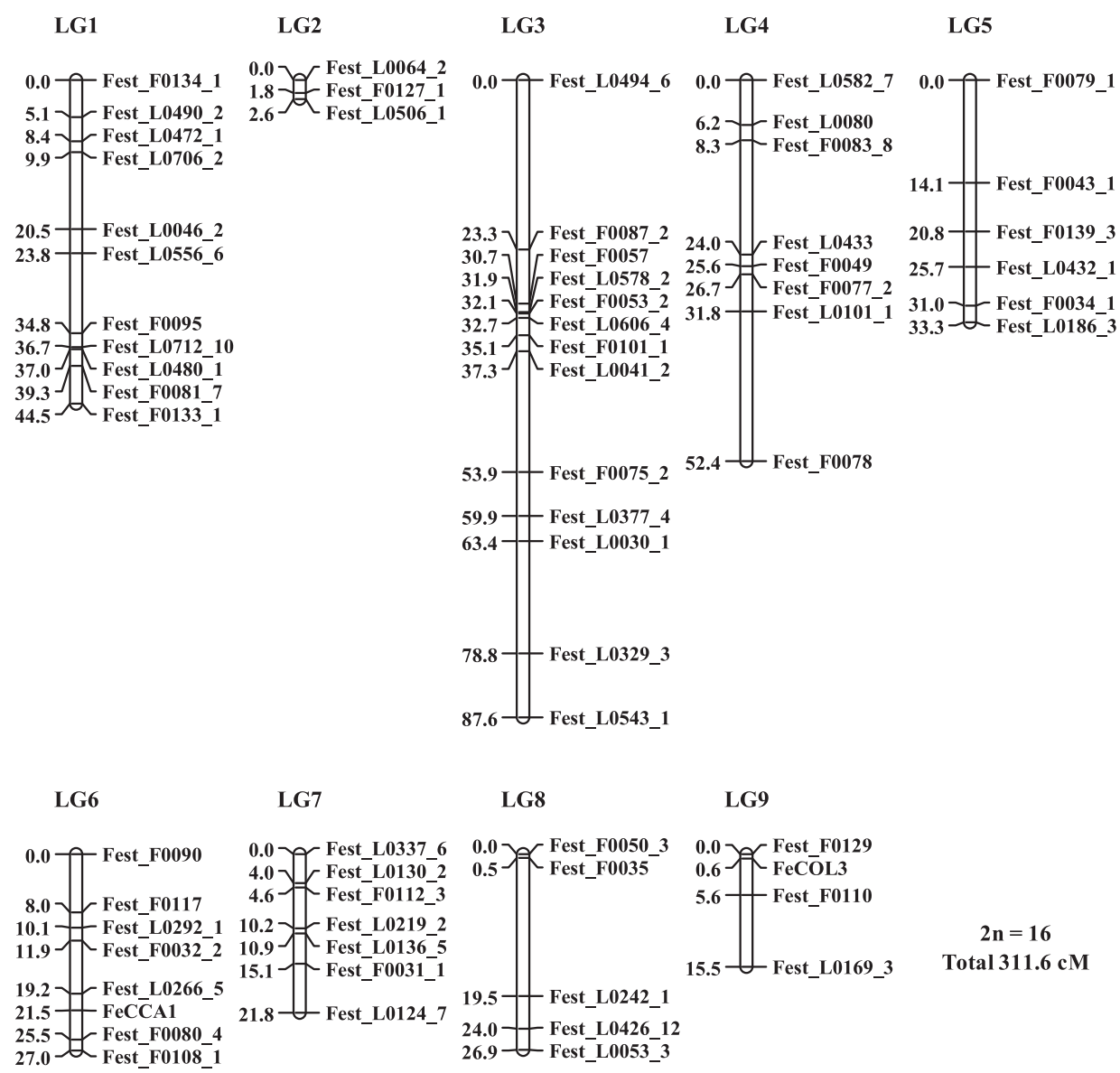

Fig. 2. Linkage map of common buckwheat.

ranging from $2.6 \mathrm{cM}(\mathrm{LG} 2)$ to $87.6 \mathrm{cM}(\mathrm{LG} 3)$ and covered $311.6 \mathrm{cM}$ in total. The segregation ratios for the three candidate gene markers were not significantly different from the expected Mendelian ratios. $\mathrm{FeCOL} 3$ and $\mathrm{FeCCA1}$ markers were mapped to LG9 and LG6, respectively, but FeELF3 could not be mapped (Table 2 and Fig. 2).

\section{QTL analysis for photoperiod sensitivity}

The segregation of photoperiod sensitivity in the $\mathrm{F}_{4}$ progenies was observed as a wide but unimodal distribution. Transgressive segregation in the direction of late flowering was observed (Fig. 3).

When we performed interval mapping, two regions were associated with photoperiod sensitivity: one in LG3 (25.3 to $50.3 \mathrm{cM}$ ) and one in LG7 (0 to $15.1 \mathrm{cM}$ ) (LOD score $>2.4$, $P<0.05)$. The results of cofactor analysis indicated a significant difference $(P<0.02)$ at eight markers (Fest_L0490_2 and Fest_L0472_1 in LG1, Fest_L0606_4 in LG3, Fest_F0077_2 and Fest_L0101_1 in LG4, Fest_L0337_6 in LG7 and Fest_L0242_1 and Fest_L0053_3 in LG8). In the MQM analysis, two QTLs (Fest_L0606 4 and Fest_L0337_6 in LG3 and 7, respectively) showed relatively high LOD values (LOD score $>2.4, P<0.05$ ) (Table 3 and Fig. 4). For each of these two QTLs, the KYU alleles had negative additive effects on days-to-flowering ( -8.2 days

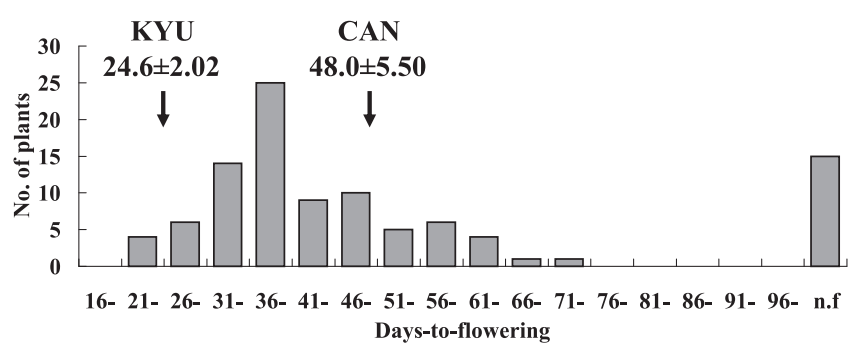

Fig. 3. Distribution of days-to-flowering in $\mathrm{F}_{4}$ progeny $(\mathrm{n}=100)$ of a cross of 02AL113(Kyukei SC2)LH.self (KYU) $\times$ C0408-0 RP (CAN). Arrows indicate means \pm SD for each parent. n.f., non-flowering.

and -6.8 days, for Fest L0606 4 and Fest L0337 6, respectively) and explained about $20.0 \%$ and $14.2 \%$ of the phenotypic variation in the $\mathrm{F}_{4}$ progenies, respectively.

In the candidate gene markers, $\mathrm{FeCCA1}$ and $\mathrm{FeCOL3}$ showed low LOD values (LOD score 0.13 and 0.11 , respectively) as a result of the MQM analysis (Table 3). FeELF3 could not be mapped. Thus, we searched for associations between FeELF3 and photoperiod sensitivity by using the ANOVA. Table 4 shows the result of the ANOVA for association between the FeELF3 and photoperiod sensitivity. It was shown that $F e E L F 3$ was significantly related to photoperiod sensitivity $(P=0.028)$. For FeELF3 markers, CAN 
Table 3. Quantitative trait loci (QTLs) detected for photoperiod sensitivity in the 02AL113(Kyukei SC2)LH.self $(\mathrm{KYU}) \times$ C0408-0 RP (CAN) $\mathrm{F}_{4}$ population by multiple QTL analysis

\begin{tabular}{lclccc}
\hline \hline LG & $\begin{array}{c}\text { Position } \\
(\mathrm{cM})\end{array}$ & Locus & LOD & $\begin{array}{c}\text { Additive } \\
\text { effect }\end{array}$ & PVE (\%) \\
\hline 3 & 34.0 & Fest_L0606_4 & 5.2 & -8.2 & 20.0 \\
7 & 0.0 & Fest_L0337_6 & 3.8 & -6.8 & 14.2 \\
6 & 21.5 & FeCCA1 & 0.13 & 1.2 & 0.4 \\
9 & 0.6 & FeCOL3 & 0.11 & -1.1 & 0.4 \\
$\mathrm{U}$ & - & FeELF3 & - & - & - \\
\hline
\end{tabular}

$\mathrm{U}$, unknown genome location

Table 4. Quantitative trait loci (QTLs) detected for photoperiod sensitivity in the 02AL113(Kyukei SC2)LH.self $(\mathrm{KYU}) \times$ C0408-0 RP (CAN) $\mathrm{F}_{4}$ population by ANOVA. KYU and CAN indicate average of days-to-flowering for the 02AL113(Kyukei SC2)LH.self allele and homozygosity for the C0408-0 RP allele, respectively.

\begin{tabular}{lclrrrr}
\hline \hline LG & $\begin{array}{c}\text { Position } \\
(\mathrm{cM})\end{array}$ & Locus & $F$ value & $P$ value & \multicolumn{3}{c}{$\begin{array}{c}\text { Average of days- } \\
\text { to-flowering }\end{array}$} \\
\cline { 5 - 7 } & & & & & KYU & CAN \\
\hline $\mathrm{U}$ & - & FeELF3 & 3.72 & 0.028 & 51.5 & 42.9 \\
3 & 34.0 & Fest_L0606_4 & 10.40 & $<0.001$ & 39.3 & 54.6 \\
7 & 0.0 & Fest_L0337_6 & 6.17 & 0.003 & 40.6 & 53.1 \\
\hline
\end{tabular}

$\mathrm{U}$, unknown genome location

allele showed smaller average of days-to-flowering than KYU allele, the difference was 8.6 days while KYU allele had early average of days-to-flowering (the difference was 15.3 and 12.5 days, for Fest_L0606_4 and Fest_L0337_6, respectively) in the two QTL's.

To test for the existence of QTL interactions, genotype data for Fest_L0606_4, Fest_L0337_6 and FeELF3 were used in a two-way ANOVA to test the phenotypic difference among the four homozygous genotype classes (Fig. 5). A significant interaction was detected between Fest_L0606_4 and Fest_L0337_6 $(P<0.01)$. On the other hand, interactions between Fest_L0606_4 and FeELF3 and between Fest_L0337_6 and $\bar{F} e E L F 3$, were not significant $(P=0.81$ and $\bar{P}=0.89$, respectively).

\section{Discussion}

A number of studies have been conducted for investigating photoperiod sensitivity in common buckwheat. Minami (1985) suggested that photoperiod sensitivity of common buckwheat is controlled by the action of multiple genes. However, neither genes nor candidate regions related to photoperiod sensitivity had been identified until now. In the present study, a difference in days-to-flowering between the KYU and CAN populations was expressed under a $15.5 \mathrm{~h}$ photoperiod, but no difference was observed under the $14.5 \mathrm{~h}$ photoperiod. These results showed that the difference in days-to-flowering between KYU and CAN observed under the $15.5 \mathrm{~h}$ photoperiod was caused by a difference of photo-
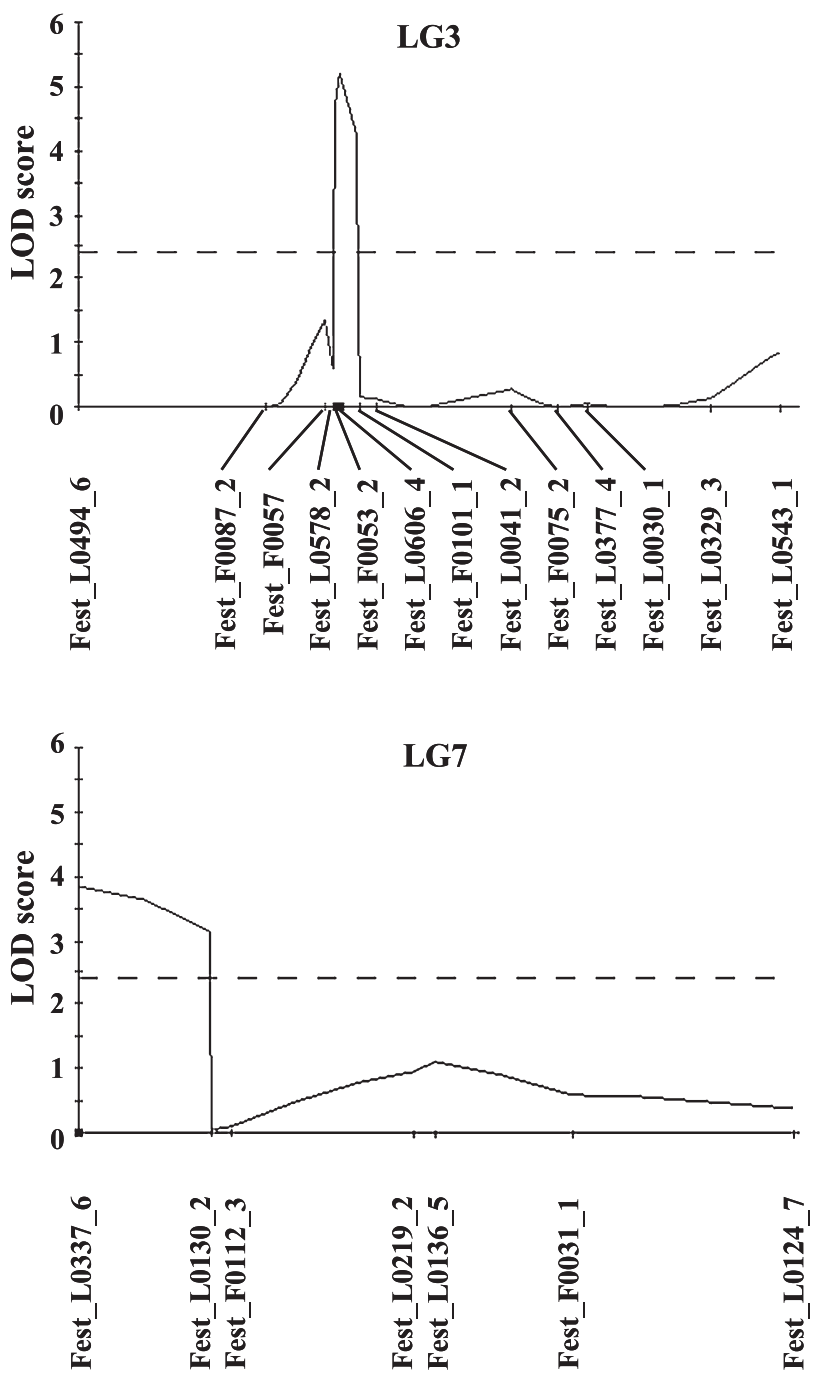

Fig. 4. LOD score for QTLs detected in multiple QTL analysis. The QTL likelihood maps for each linkage group were obtained by using the MQM procedure of MapQTL, fixing one marker cofactor per putative QTL. Selected markers used as cofactors in analysis are indicated by black rectangles along the $x$-axes. The linkage group number is indicated at the top of each graph. Horizontal dashed lines indicate the $2.4(P<0.05)$ LOD score threshold.

period sensitivity: the KYU population had weaker photoperiod sensitivity than the CAN population. The KYU line was bred by interspecific hybridization among $F$. homotropicum (an autogamous species) and F. esculentum cv. Botansoba which is cultivated in high-latitude regions (Matsui 2006); similarly, the CAN line was also bred by crossing $F$. homotropicum and $F$. esculentum which is cultivated in high-latitude regions (Wang and Campbell 1998). Thus, we assumed that both lines had been selected under long-day conditions. This may be why the difference in photoperiod sensitivity between KYU and CAN was not evident unless an artificially long day length (15.5 h) was applied.

In Arabidopsis, the photoperiod-sensitivity pathway consists of photoreceptors, the circadian clocks, and floral 

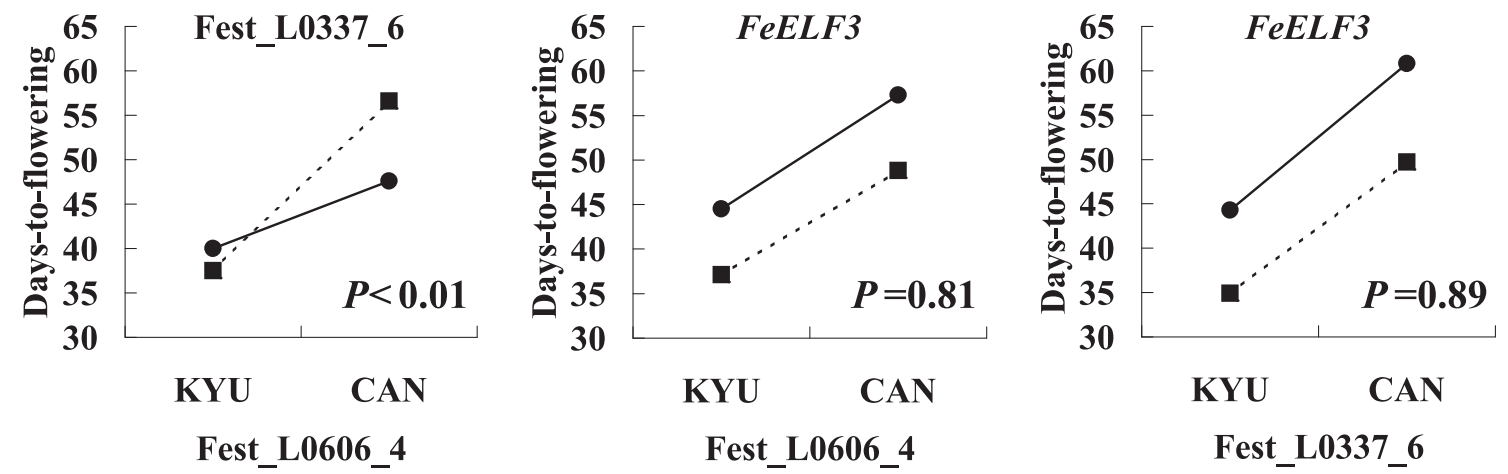

Fig. 5. Mean values for days-to-flowering in different combinations of genotype classes for Fest_L0606_4 and Fest_L0337_6. The $x$-axis indicates the genotype at Fest_L0606-4 and Fest_L0337_6: KYU and CAN indicate homozygosity for the 02AL113(Kyukei SC2)LH.self allele and homozygosity for the C0408-0 RP allele, respectively. The genotype of Fest L0337 6 and FeELF3 are indicated by circles (homozygous for KYU allele) or squares (homozygous for CAN allele). $P$ value in each figure showed results of significance for interaction by two way ANOVA.

promoters (Putterill et al. 2004). In many species with photoperiod sensitivity, such as rice, wheat (Triticum aestivum), and morning glory (Pharbitis nil), genes orthologous to photoperiod-sensitivity genes found in Arabidopsis were confirmed to be related to heading date or flowering time (Hayama et al. 2002, 2003, Kojima et al. 2002, Liu et al. 2001a, Nemoto et al. 2003, Yano et al. 2000). In the present study, we found three such candidate gene regions ( $\mathrm{FeCOL3}, \mathrm{FeCCA1}$ and $\mathrm{FeELF3}$ ) among 863 cDNA clones. In Arabidopsis, CCA1 and ELF3 are genes that connect the circadian clock to the photoperiod-sensitivity pathway (Covington et al. 2001, Hicks et al. 2001, Liu et al. 2001b, Wang et al. 1997, Wang and Tobin 1998), and COL3 is from a $\mathrm{CO}$-related gene family that connects floral promoters to the photoperiod-sensitivity pathway (Putterill et al. 1995). Here, we found that the FeELF3 candidate gene region in common buckwheat was strongly associated with photoperiod sensitivity. Although the candidate genes identified in the present study would only be part of the total group of genes included in a photoperiod-sensitivity pathway, our report is the first in which candidate genes identified based on their role in photoperiod sensitivity in another species were associated with photoperiod sensitivity in common buckwheat. The results indicate that it would be efficient to study the photoperiodsensitivity pathway by using other candidate genes and analyzing their effects on photoperiod sensitivity. Although mutations in intron region were reported to be associated with gene expression (Fiume et al. 2004, Fu et al. 2005, Isshiki et al. 1998, Samadder et al. 2008), it was not clear whether FeELF3 marker based on SNP in an intron region directly associated with photoperiod sensitivity right now. If SNP's associated with photoperiod sensitivity in direct will be detected in the future, the allelic variations in FeELF3 gene region will become more informative information.

In the MQM analysis, we detected two QTLs, Fest_L0606_4 and Fest_L0337_6, which explained about $20.0 \%$ and $\overline{14} .2 \%$ the variation observed in the $\mathrm{F}_{4}$ progenies, respectively. In the present study, we constructed a linkage map for QTL analysis consisting of 9 linkage groups con- taining 63 ESTs and two candidate gene regions and covering $311.6 \mathrm{cM}$ in total. This map was the first linkage map to include ESTs and candidate gene regions thought to be related to photoperiod sensitivity in common buckwheat. Unfortunately, we assumed that the genome coverage of this map is not so high because Konishi and Ohnishi (2006) constructed a linkage map of buckwheat covering about $900 \mathrm{cM}$ by using AFLP and SSR markers. Thus, result of MQM analysis at unmapped $\mathrm{FeELF} 3$ candidate gene region was not shown. If the genome coverage of this map will be higher, other QTLs included FeELF3 candidate gene region might be detected.

The genetic analysis of quantitative traits with molecular markers makes it possible to evaluate epistatic interactions between individual loci. A digenic interaction between the QTLs detected in the MQM analysis was observed in this study. The additive effect of the KYU allele of Fest L0337_6 was estimated to be -6.8 days. However, the observed two-locus interaction between Fest_L0606 4 and Fest_L0337_6 suggests that the effects of Fest_L0337_6 are changed depending on the allele present at Fest_L0606_4. In Arabidopsis and rice, interactions between genes related to photoperiod sensitivity have been reported (Putterill et al. 2004, Yano et al. 1997). These results suggested that photoperiod sensitivity in common buckwheat was controlled by both main and interaction effects of the QTLs detected. Thus, it is necessary to analyze both the main effects and the interactions between QTLs to fully understand photoperiod sensitivity.

In summary, our study is the first report that photoperiod sensitivity in common buckwheat is controlled by the action of multiple genes, including interaction effects between these genes. The photoperiod sensitivity was controlled by at least three loci, including candidate genes identified as being part of the photoperiod-sensitivity pathway in Arabidopsis. These findings will serve as a stepping-stone to future studies on the role of photoperiod sensitivity in local adaptation and ecological breeding in common buckwheat. 


\section{Acknowledgments}

We thank Dr. J. Aii at the Niigata University of Pharmacy and Applied Life Sciences for providing the Fest_F cDNA clones. We also thank Dr. C. Campbell at the Kade Research Ltd. for providing seeds of the C0408-0RP. This study was partly supported by the Japanese Society for the Promotion of Science via a Grant-in-Aid for Scientific Research (B) (16380003).

\section{Literature Cited}

Churchill,G.A. and R.W.Doerge (1994) Empirical threshold values for quantitative trait mapping. Genetics 138: 963-971.

Corbesier,L. and G.Coupland (2005) Photoperiodic flowering of Arabidopsis: integrating genetic and physiological approaches to characterization of the floral stimulus. Plant Cell Environ. 28: 54 66.

Covington, M.F., S.Panda, L.X.Liu, A.C.Strayer, R.D. Wagner and A.S.Kay (2001) ELF3 modulates resetting of the circadian clock in Arabidopsis. Plant Cell 13: 1305-1315.

Fiume,E., P.Christou, S.Giani and D.Breviario (2004) Introns are key regulatory elements of rice tubulin expression. Planta 218: 693703.

Fu,D., P.Szucs, L.Yan, M.Helguera, J.S.Skinner, J.von Zitzewitz, P.M.Hayes and J.Dubcovsky (2005) Large deletions within the first intron in $V R N-1$ are associated with spring growth habit in barley and wheat. Mol. Gen. Genomics 273: 54-65.

Gordon,D.A.C. and P.Green (1998) Consed: A graphical tool for sequence finishing. Genome Res. 8: 195-202.

Hayama,R., T.Izawa and K.Shimamoto (2002) Isolation of rice genes possibly involved in the photoperiodic control of flowering by a fluorescent differential display method. Plant Cell Physiol. 43: 494-504.

Hayama,R., S. Yokoi, M.Yano and K. Shimamoto (2003) Adaptation of photoperiodic control pathways produces short-day flowering in rice. Nature 422: 719-722.

Hayama, R. and G.Coupland (2003) Shedding light on the circadian clock and the photoperiodic control of flowering. Curr. Opin. Plant Biol. 6: 13-19.

Hicks, K.A., M.T. Albertson and R.D. Wagner (2001) EARLY FLOWERING3 encodes a novel protein that regulates circadian clock function and flowering in Arabidopsis. Plant Cell 13: 12811292.

Isshiki,M., K.Morino, M.Hakajima, R.J.Okagaki, S.R.Wessler, T. Izawa and K. Shimamoto (1998) A naturally occurring functional allele of the rice waxy locus has a GT to TT mutation at the $5^{\prime}$ splice site of the first intron. Plant J. 15: 133-138.

Iwata,H., K.Imon, Y.Tsumura and R.Ohsawa (2005) Genetic diversity of common buckwheat varieties in Japan based on microsatellite markers. Genome 48: 367-377.

Jansen,R.C. (1993) Interval mapping of multiple quantitative trait loci. Genetics 135: 205-211.

Jansen, R.C. and P.Stam (1994) High resolution of quantitative traits into multiple loci via interval mapping. Genetics 136: 1447-1455.

Kojima,S., Y.Takahashi, Y.Kobayashi, L.Monna, T.Sasaki, T.Araki and M.Yano (2002) Hd3a, a rice ortholog of the Arabidopsis FT gene, promotes transition to flowering downstream of $\mathrm{Hd} 1 \mathrm{under}$ short-day conditions. Plant Cell Physiol. 43: 1096-1105.

Konishi,T. and O.Ohnishi (2006) A linkage map for common buck- wheat based on microsatellite and AFLP markers. Fagopyrum 23: $1-6$.

Lander,E.S. and D.Botstein (1989) Mapping Mendelian factors underlying quantitative traits by using RFLP linkage maps. Genetics 121: 1447-1455.

Liu,J., J.Yu, L.McIntosh, H.Kende and J.A.D.Zeevaart (2001a) Isolation of a CONSTANS ortholog from Pharbitis nil and its role in flowering. Plant Physiol. 125: 1821-1830.

Liu,X.L., F.M.Covington, C.Fankhauser, J.Chory and R.D.Wagner (2001b) ELF3 encodes a circadian clock-regulated nuclear protein that functions in an Arabidopsis PHYB signal transduction pathway. Plant Cell 13: 1293-1304.

Matano,T. and A.Ujihara (1979) Agroecological classification and geographical distribution of the common buckwheat, Fagopyrum esculentum M. in the East Asia. JARQ 13: 157-162.

Matsui,K. (2006) A genetic and breeding study for the production of self-compatible buckwheat cultivars (Self-compatible buckwheat cultivars). Bulletin of the National Agricultural Research Center for Kyushu Okinawa Region. 47: 1-42.

Minami,H. (1985) Ecological-genetic studies of ecological differentiation in common buckwheat. Doctoral thesis, University of Tsukuba, p. 62.

Minami,H. and H. Namai (1986a) Potential genetic variation of flowering time in late-summer type cultivar of buckwheat (Fagopyrum esculentum Moench) in Kyushu region [Japan]. Jpn. J. Breed. 36: $67-74$.

Minami,H. and H.Namai (1986b). Populational change in flowering time caused by different harvesting date observed in the latesummer type cultivar Miyazakizairai of buckwheat (Fagopyrum esculentum). Jpn. J. Breed. 36: 155-162.

Nagatomo,T. (1961) Studies on physiology of reproduction and some cases of inheritance in buckwheat. Research Report of Plant Breeding Laboratory in University of Miyazaki 1: 1-213.

Nakamura, M. and H.Nakayama (1950) On the enervative sterility in buckwheat. Japan. Jour. Crop Sci. 19: 122-125.

Namai,H. (1990) Pollination biology and reproductive ecology for improving genetics and breeding of common buckwheat, Fagopyrum esculentum (1). Fagopyrum 10: 23-46.

Neff,M.M., E.Turk and M.Kalishman (2002) Web-based primer design for single nucleotide polymorphism analysis. Trends Genet. 18: 613-615.

Nemoto,Y., M.Kisaka, T.Fuse, M.Yano and Y.Ogihara (2003) Characterization and functional analysis of three wheat genes with homology to the CONSTANS flowering time gene in transgenic rice. Plant J. 36: 82-93.

Ohsawa,R. (1997) Evaluation of a Japanese germplasm collection of common buckwheat using a multivariate approach. Proceedings of the 8th SABRAO Congress, pp. 107-108.

Putterill,J., F.Robson, K.Lee, R.Simon and G.Coupland (1995) The CONSTANS gene of Arabidopsis promotes flowering and encodes a protein showing similarities to zinc finger transcription factors. Cell 80: 847-857.

Putterill,J., R.Laurie and R.Macknight (2004) It's time to flower: the genetic control of flowering time. Bioessays 26: 363-373.

Samadder,P., E.Sivamani, J.Lu, X.Li and R.Qu (2008) Transcriptional and post-transcriptional enhancement of gene expression by the $5^{\prime}$ UTR intron of rice rubi3 gene in transgenic rice cells. Mol. Genet. Genomics 279: 429-439.

Sugawara,K. (1958) On the injury of buckwheat pistil, Retardation of pistil growth as influenced by day-length. Japan. Jour. Crop Sci. 26: 269-270. 
Van Ooijen,J.W. (2005) Map-QTL_5: software for the mapping quantitative trait loci in mapping populations. Kyazma BV, Wageningen

Van Ooijen,J.W. (2006) JoinMap, Software for the Calculation of Genetic Linkage Maps (Kyazma BV, Wageningen, The Netherlands), Version 4.

Wang, Y.J. and C.Campbell (1998) Interspecific hybridization in buckwheat among Fagopyrum esculentum, F. homotropicum and F. tataricum. Proc 7th Int Symp Buckwheat at Winnipeg, Canada. I: $1-12$.

Wang,Z.Y., D.Kenigsbuch, L.Sun, E.Harel, S.M.Ong and M.E.Tobin (1997) A Myb-related transcription factor is involved in the phytochrome regulation of an Arabidopsis Lhcb gene. Plant Cell 9: 491507.

Wang,Z.Y. and M.E.Tobin (1998) Constitutive expression of the
CIRCADIAN CLOCK ASSOCIATED 1 (CCA1) gene disrupts circadian rhythms and suppresses its own expression. Cell 93: 1207-1217.

Yano, M., Y.Harushuma, Y.Nagamura, N. Kurata, Y.Minobe and T.Sasaki (1997) Identification of quantitative trait loci controlling heading date in rice using a high-density linkage map. Theor. Appl. Genet. 95: 1025-1032.

Yano,M., Y.Katayose, M.Ashikan, U.Yamanouchi, L.Monna, T.Fuse, T.Baba, K. Yamamoto, Y.Umehara, Y.Nagamura et al. (2000) $H d 1$, a major photoperiod sensitivity quantitative trait locus in rice, is closely related to the Arabidopsis flowering time gene CONSTANS. Plant Cell 12: 2473-2483.

Yano,M., S.Kojima, Y.Takahashi, H.Lin and T.Sasaki (2001) Genetic control of flowering time in rice, a short-day plant. Plant Physiol. 127: 1425-1429. 